\title{
A METHOD FOR MONITORING BULGE OF ANCIENT CITY WALL AFTER REPAIR
}

\author{
Baicheng Feng ${ }^{1} \cdot 2 *$, Yungang $\mathrm{Hu}^{1,2}$, Miaole $\mathrm{Hou}^{1,2}$ \\ ${ }^{1}$ School of Geomatics and Urban Spatial Informatics, Beijing University of Civil Engineering and Architecture, No.15 Yongyuan \\ Road, Daxing District, Beijing; 102616, China; 2108160120003@stu.bucea.edu.cn (B.F.); huyungang@bucea.edu.cn (Y.H.); \\ houmiaole@bucea.edu.cn (M.H.). \\ ${ }^{2}$ Beijing Key Laboratory for Architectural Heritage Fine Reconstruction and Health Monitoring, No.15 Yongyuan Road, Daxing \\ District, Beijing; 102616, China
}

KEY WORDS: Heritage protection, City wall diseases, Bulge extraction, Coordinate transformation, Deformation monitoring.

\begin{abstract}
:
The ancient city wall is affected by factors such as environment and man-made factors, and the formed bulging disease is particularly prominent, and even caused irreversible damage to the ancient city wall. Therefore, the bulging monitoring after the repair of the city wall should be the top priority of the deformation monitoring of the city wall. This paper proposes a new plan for monitoring the bulge after the repair of the city wall. According to the point cloud data before the repair, the characteristic plane is fitted to determine the bulge range of the city wall, and then observation signs are placed on the surface of the repaired city wall at the corresponding location where the bulging deformation is severe. By using a total station to monitor the space coordinates of the observation signs, the deformations perpendicular to the wall can be obtained through coordinate conversion, and then the bulging of the repaired city wall can be determined. The actual application results show that this method can effectively monitor the swelling of the ancient city wall after the repair.
\end{abstract}

\section{INTRODUCTION}

As the framework of ancient Chinese cities, the ancient city wall reflects the construction technology and ideological form of the corresponding dynasty under the requirements of military defence and ancient city planning, and reflects the level of ancient Chinese cultural development on the side. It is known as "the most basic of Chinese cities. The most impressive and durable part"(Oscald, 2017), is one of the important ancient buildings. However, due to years of wind and rain erosion, plant growth and other natural conditions, as well as the intervention of human factors, many ancient city walls have suffered severe damage.

Regarding the protection of the city wall, Mr. Liang Sicheng put forward the concept of "City Wall Three-dimensional Park". After decades of development, the form of the city wall park has been effectively promoted and utilized, and the city wall has been effectively protected( $\mathrm{Li}, 2020)$. However, the protection measures based on the monitoring of the city wall itself can obtain the deformation state of the city wall more accurately. The bulging disease of the city wall is one of the main problems of the city wall, as shown in Figure 1. Based on the principle of preventive protection of ancient buildings, the bulging monitoring after the repair of the city wall will be a prominent focus of the city wall deformation monitoring.

At present, there is a lack of specific research on the method of monitoring the bulge after the city wall is repaired. The general monitoring method is to evenly distribute the observation signs on the city wall according to the distance, and calculate the deformation by comparing the monitoring data (Zhou, 2011). However, this method cannot cover the key monitoring range and is due to the observation signs. The falling off and deformation of the swelling and deformation caused the inaccuracy of the swelling deformation data (Li, 2018). In response to this situation, this article proposes a new monitoring method that can realize the monitoring of key swollen disease areas, and through the new observation signs designed for monitoring, it can be realized from the two aspects of accuracy requirements and ancient building protection principles. Effective monitoring of swelling after the ancient city wall is repaired
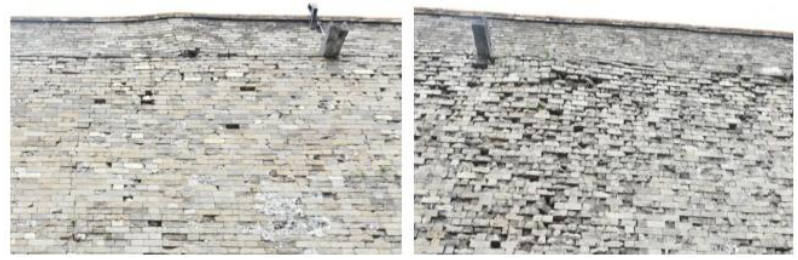

Figure 1. Bulge of the ancient wall

\section{THE BASIC IDEA}

The monitoring after the completion of the repair of the city wall is divided into three steps: determining the area with severe bulging, placing observation signs, and performing point monitoring and data processing.

Before repairing, perform 3D laser scanning on the whole ancient city wall, first preprocess the point cloud data, reduce the influence of noise on the data, and complete the fine model of the city wall (Kong, 2019). The point cloud data is fitted with a plane, and the fitted plane is compared with the established model. Take a point on the plane and find its normal vector, then the distance from any point on the model to the plane is the projection length of the connection line between the plane point and the model point on the plane point normal vector, that is, the deviation from each point to the fitted plane, this deviation is regarded as the size of the bulging value. The above method is used to determine the distance from the point on the model to the fitting plane, and different thresholds are adjusted to determine the swelling area with different swelling values.

After the repair of the city wall is completed, observation signs will be placed in the more serious bulging area to monitor the bulge after the repair of the city wall is completed. When using a total station to monitor the external swelling and deformation of the wall, the observation signs should be distributed as evenly or

* Corresponding author: E-mail address: 2108160120003@stu.bucea.edu.cn 
targeted as possible in the key monitoring area, so as to analyse the swelling and deformation laws of the city wall at different locations in the later stage.

After setting up the corresponding observation signs, the total station can be used to measure the three-dimensional space coordinates of the points. Finally, in order to accurately obtain the deformations perpendicular to the city wall, the station coordinate system needs to be converted to the city wall coordinate system. Through the conversion of seven parameters in the space coordinate system, the difference of the results of the coordinate conversion of the monitoring data after the two phases of coordinate conversion is compared, and the deformation perpendicular to the wall surface, that is, the amount of swelling change, can be obtained. Through multi-period monitoring, it is possible to accurately learn the bulging difference and the total bulging that occurred at each monitoring point during the monitoring cycle.

Summarize the swelling difference of each monitoring point, and further add temperature and humidity sensors outside the city wall to study the correlation between swelling and temperature and humidity, and try to analyse the main environmental factors that affect the swelling of the city wall.

\section{DATA ACQUSITION}

The monitoring of an ancient city wall with the above method is now carried out. Before the repairs start, a three-dimensional laser scanner is used to collect data on the city wall. After processing the original data, according to the degree of swelling of the city wall, an appropriate swelling value is taken as the lowest threshold for swelling extraction, which is usually 1 to 20 $\mathrm{cm}$. Defining the extraction range, determining the bulging area generated by different deviations, and extracting the contour line of the bulging area. Through the extracted contour lines, the key monitoring area for bulging can be determined.

After the city wall is repaired, place observation signs in key monitoring areas. The following three parts need to be considered when laying observation signs: 1 . Try to evenly cover key monitoring areas with similar swelling amounts. 2 . The swelling amount that may be caused by the different positions of the city wall should be considered when laying, in order to facilitate the subsequent analysis of the correlation between the swelling of the city wall and the location. 3 . For the protection and ornamental principles of ancient building monitoring, it is not suitable to damage the bricks after the repair of the ancient city wall, nor to be unsightly in appearance.

The observation sign adopts a newly designed wall joint fixed prism mounting bracket, which includes a $360^{\circ}$ small prism, a rain shield and a prism pole. The prism pole is inclined, and the upper end of the prism pole is connected with the prism, including the wall gap fixed inserts and supporting columns can be fixed in the wall joints. The overall appearance of the observation sign is shown in Figure 2. According to the results of the comparison experiment between the $360^{\circ}$ small prism and the ordinary prism, the measurement result of the $360^{\circ}$ small prism fully meets the accuracy requirements.

Each key monitoring area is laid out in the form shown in Figure 3. During the repair of the city wall, fixed inserts are inserted between the gaps in the wall tiles. One group is placed in each monitoring area, and a total of three groups of observation signs are laid. The group contains five observation signs. The distance between the observation signs is based on the size of the bulging area. The vertical distance is about $2.1 \mathrm{~m}$ along the city wall, and the horizontal distance is about $1.5 \mathrm{~m}$ along the city wall.

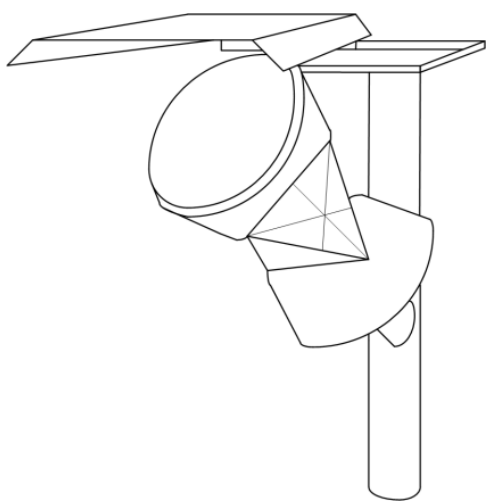

Figure 2. Schematic diagram of observation signs

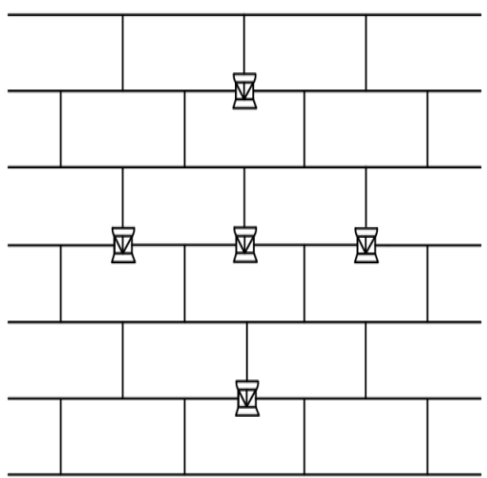

Figure 3. Layout scheme of observation signs

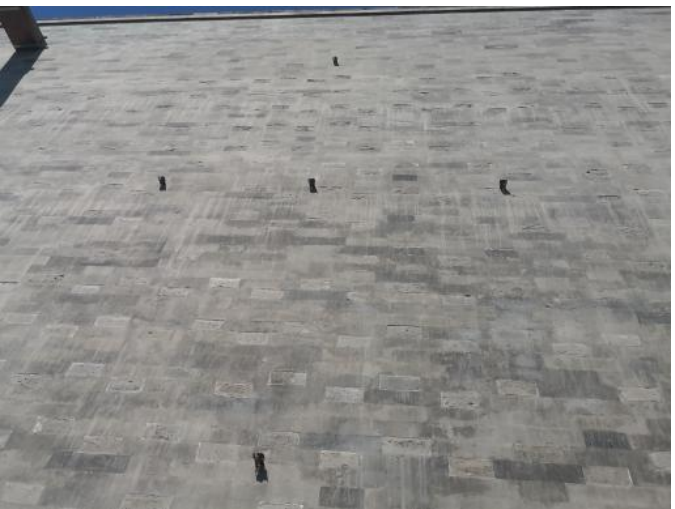

Figure 4. Layout of observation signs

In order to determine the accuracy of the measurement data to the greatest extent and reduce the error caused by the erection of the measuring station, two obligatory centring observation piers were buried before the formal bulging monitoring was carried out. One is used as a measuring station and the other is used for orientation. During each monitoring process, the coordinates of the two observation piers need to be checked to ensure the stability of the observation pier. Using the total station to directly measure the space coordinates of the observation mark, the coordinates of the observation mark in the station coordinate system can be obtained. Since there is an inclination angle of about $82^{\circ}$ between the surface of the city wall and the ground, it is not strictly vertical to the ground, and in the horizontal direction, it is difficult to establish a mandatory centring 
observation pier to be strictly parallel to the horizontal direction of the city wall. Therefore, the two phases are under the station coordinate system The coordinate difference obtained by direct monitoring cannot accurately reflect the size of the swelling of the observation mark perpendicular to the surface of the city wall during the monitoring process, so it is necessary to convert the station coordinate system to the city wall coordinate system. The city wall coordinate system requires the $\mathrm{X}$ axis to be parallel to the city wall and the $\mathrm{Y}$ axis to be perpendicular to the city wall surface. The conversion diagram is shown in Figure 4.

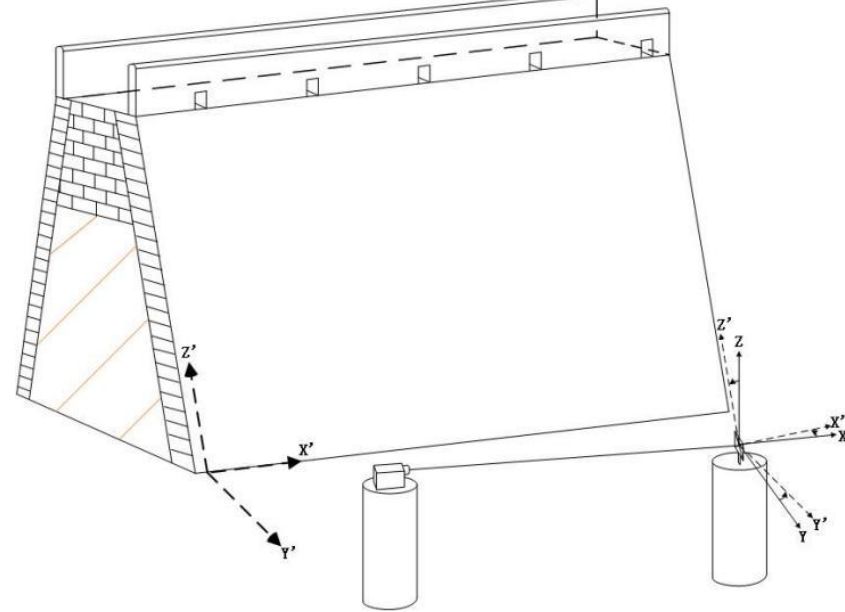

Figure 5.Coordinate conversion diagram

By laying at least three reflectors on the city wall, the spatial coordinates of the reflectors in the two coordinate systems are obtained by using methods such as total station and steel ruler distance measurement, taking into account the accuracy of the coordinate conversion result from the rotation angle in the coordinate conversion The least square method of Rodriguez matrix applicable to any rotation angle is adopted to realize coordinate conversion (Han, 2016).

Suppose the coordinates of a point on the wall in the space rectangular coordinate system $O-X Y Z$ and $O-X^{\prime} Y^{\prime} Z^{\prime}$ are $(X, Y, Z)$ and $\left(X^{\prime}, Y^{\prime}, Z\right)$ respectively, $a_{1}, a_{2}, a_{3}$ is the cosine of the angle between axis $X^{\prime}$ and axis $X, Y, Z ; b_{1}, b_{2}, b_{3}$ is the cosine of the angle between axis $Y^{\prime}$ and axis $X, Y, Z, c_{1}, c_{2}, c_{3}$ is the axis The cosine of the angle between $Z^{\prime}$ and axis $X, Y, Z$, $\lambda$ is the scale parameter; $(\Delta X, \Delta Y, \Delta Z)$ is the translation parameter, the relationship is as follows:

$$
\left[\begin{array}{l}
x \\
y^{\prime} \\
z^{\prime}
\end{array}\right]=\lambda\left[\begin{array}{lll}
a_{1} & a_{2} & a_{3} \\
b_{1} & b_{2} & b_{3} \\
c_{1} & c_{2} & c_{3}
\end{array}\right]\left[\begin{array}{l}
x \\
y \\
z
\end{array}\right]+\left[\begin{array}{l}
\Delta x \\
\Delta y \\
\Delta z
\end{array}\right]
$$

First introduce an antisymmetric matrix:

$$
S=\left[\begin{array}{ccc}
0 & -c & -b \\
c & 0 & -a \\
b & a & 0
\end{array}\right]
$$

Then $R=(I+S)(I-S)^{-1}$ is an orthogonal matrix, expand it, this orthogonal matrix is Rodriguez matrix. When Rodriguez matrix is used to express the direction cosine matrix, the required conversion parameter is $\lambda, a, b, c, \Delta x, \Delta y, \Delta z$. The parameter calculation process can be divided into 3 steps, first find the scale parameter, then the rotation parameter, and finally the translation parameter.

The scale factor is:

$$
\lambda=\frac{\sqrt{\left(x_{2}^{\prime}-x_{1}^{\prime}\right)^{2}+\left(y_{2}^{\prime}-y_{1}^{\prime}\right)^{2}+\left(z_{2}^{\prime}-z_{1}^{\prime}\right)^{2}}}{\sqrt{\left(x_{2}-x_{1}\right)^{2}+\left(y_{2}-y_{1}\right)^{2}+\left(z_{2}-z_{1}\right)^{2}}}
$$

From the error equation:

$$
V=\underset{3(n-1) \times 33 \times 1}{B}-\underset{3(n-1) \times 3}{L}
$$

Where:

$$
\begin{gathered}
B=\left[\begin{array}{ccc}
0 & -\lambda z_{21}-z_{21}^{\prime} & -\lambda y_{21}-y_{21}^{\prime} \\
-\lambda z_{21}-z_{21}^{\prime} & 0 & \lambda x_{21}+x_{21}^{\prime} \\
\lambda y_{21}+y_{21}^{\prime} & \lambda x_{21}+x_{21}^{\prime} & 0 \\
\vdots & \vdots & \vdots \\
0 & -\lambda z_{n 1}-z_{n 1}^{\prime} & -\lambda y_{n 1}-y_{n 1}^{\prime} \\
-\lambda z_{n 1}-z_{n 1}^{\prime} & 0 & \lambda x_{n 1}+x_{n 1}^{\prime} \\
\lambda y_{n 1}+y_{n 1}^{\prime} & \lambda x_{n 1}+x_{n 1}^{\prime} & 0
\end{array}\right] \\
L=\left[\begin{array}{ccc}
x_{21}^{\prime}-\lambda x_{21}, y_{21}^{\prime}-\lambda y_{21}, z_{21}^{\prime}-\lambda z_{21}, \cdots, \\
x_{n 1}^{\prime}-\lambda x_{21}, y_{n 1}^{\prime}-\lambda y_{n 1}, z_{n 1}^{\prime}-\lambda z_{n 1}
\end{array}\right]
\end{gathered}
$$

among them:

$$
u_{i j}=u_{i}-u_{j}
$$

Solve the unknowns according to the principle of least squares:

$$
X=\left(A^{T} A\right)^{-1} A^{T} L
$$

After calculating $a, b, c$, you can find the rotation matrix, and then solve the translation parameters as follows:

$$
\left[\begin{array}{l}
\Delta x \\
\Delta y \\
\Delta z
\end{array}\right]=\left[\begin{array}{l}
x_{i}^{\prime} \\
y_{i}^{\prime} \\
z_{i}^{\prime}
\end{array}\right]-\lambda R\left[\begin{array}{l}
x_{i} \\
y_{i} \\
z_{i}
\end{array}\right]
$$

In summary, the seven parameters of coordinate conversion can be obtained, and the conversion between the station coordinate system and the city wall coordinate system can be realized. The $\mathrm{X}$ axis of the transformed coordinate system is parallel to the horizontal direction of the city wall, the $\mathrm{Y}$ axis is perpendicular to the surface of the city wall, and the $\mathrm{Z}$ axis is vertical along the down direction of the city wall. The difference of the monitoring data after the coordinate conversion of the two adjacent periods can get the swelling value of the observation mark perpendicular to the surface of the city wall.

Temperature and humidity monitoring is mainly periodic monitoring. Since the monitoring cycle of this project starts, monitoring can be realized remotely. The temperature and humidity data of the external environment of the city wall are collected at a frequency of once every 5 minutes.

\section{DATA ANALYSIS}

Using the vectorized results of the Lama Temple and its surrounding buildings and combining the height data (Figure 7), the Lama Temple and its surrounding buildings are stretched into a three-dimensional model (Figure 8) to provide a data basis for subsequent experiments.

A total of three key monitoring areas have been set up for the ancient city wall to be monitored. Now several observations are made on the observation signs of one group of monitoring areas, and each observation is about 30 days apart.

This group of observation signs includes five observation signs, named after JC01, JC02, JC03, JC04, JC05. The monitoring difference data of the previous eight periods is taken as an example. The relationship between the cumulative bulging deformation of the five prisms and the number of monitoring periods is shown in the figure 5. The change trend of the overall monitoring data is negative growth. Among them, the cumulative swelling change of the JC02 prism is the largest. The cumulative deformation of the eight-time monitoring is $-4.0 \mathrm{~mm}$, that is, the cumulative swelling is $4.0 \mathrm{~mm}$ inward swelling. The five prisms generally showed an inward bulging trend. Only the four prisms of JC02 and JC03 showed a different trend than other prisms. The other periods of observation showed that each prism showed a consistent bulging trend.

Take the average value of the swelling deformation difference between the JC15 observation sign closest to the temperature and humidity sensor and the temperature and humidity data monitored on the same day, and analyse the correlation. Now take 
the monitoring data of the first fifteen periods for analysis, and the humidity monitoring value is in Y with JC15. The coordinate measurement value in the direction is shown in Figure 6. Through Pearson correlation analysis and two-tailed test, the correlation between the environmental temperature and the swelling change at the $\mathrm{JC} 15$ observation mark is not significant, and the $\mathrm{P}$ between the humidity and the swelling observation value at JC15 is 0.024 , and the Pearson correlation is -0.579 , indicating that there is a significant negative correlation between the two groups of measurements. Therefore, it can be preliminarily determined that humidity is one of the important factors affecting the swelling of the city wall.

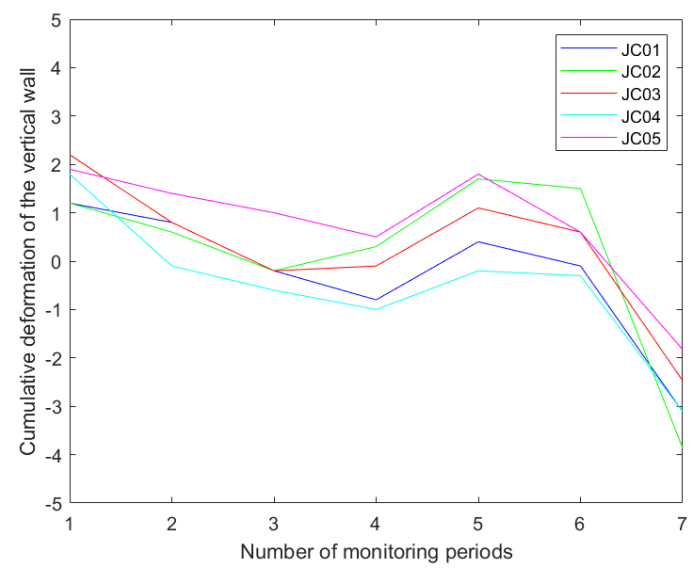

Figure 6. Schematic diagram of bulging cumulative deformation

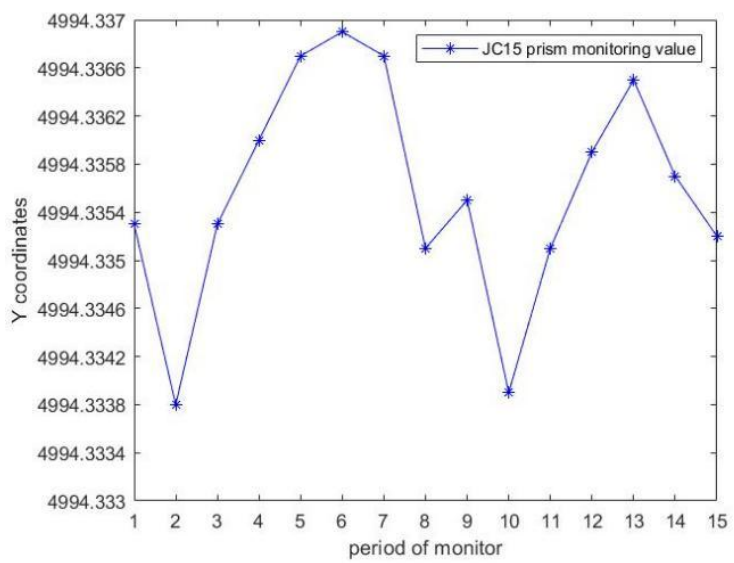

Figure 7. JC15 bulge monitoring value

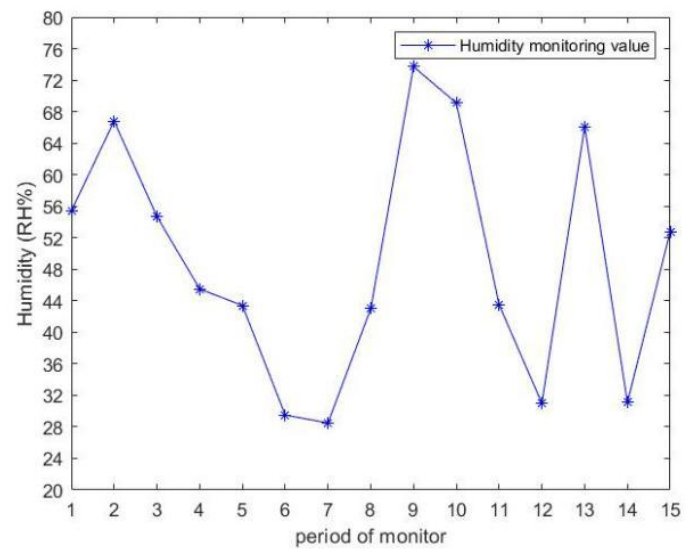

Figure 8. Humidity monitoring value

\section{CONCLUSION}

The bulging deformation of the repaired city wall is usually small. The monthly deformation is usually in the millimetre or even submillimetre level. Three-dimensional laser scanning is used to determine the severe bulging range of the city wall before the repair, and then the small fixed prism in the wall joint is used as an observation sign. Obtaining the three-dimensional space coordinates through the total station not only ensures that the surface of the repaired city wall is not damaged during the monitoring process, but also meets the accuracy of the city wall monitoring.

The results obtained show that the total deformation accumulation of the same group of observation signs is not much different, and the measured swelling amount is consistent with the actual situation. Therefore, this method can be applied to long-term ancient city wall swelling monitoring to perform a deeper measurement, except for temperature. In addition to the humidity sensor, it can be combined with equipment such as the internal structure sensor of the ancient city wall and infrared cameras to construct a multi-source data monitoring wall swelling monitoring system to further determine the cause of the wall swelling and preventive protection.

\section{ACKNOWLEDGEMENTS}

The author will thank National Key R\&D Program of China (NO. 2019YFC1520804)and Youth Beijing Scholar Program.

\section{REFERENCES}

Oscald, S., 2017. The walls and gates of Peking. Chengdu: Sichuan People's Press.

Li, Y.Y., 2020. A study on Kaifeng City Wall Park. Henan University.

Li, W., 2018 Monitoring and analysis of wall bulging of Fengyang Drum Tower. Shanxi Architecture,44(01):199-200.

Zhou, J., Hu, W.S., Zhu, M.C., 2011, Modern Surveying and Mapping,34(05):11-12+17.

Han, M.Z., Li, K.Z., 2016. Spatial Coordinate Transformation Based on Rodrigue Matrix. Engineering of Surveying and Mapping,25(04):25-27.

Kong, L., Wang, Y.C., Zhou, M.L., et al.2019.Robustness fitting method of point cloud plane based on random sampling and eigenvalue method. Geomatics \& Spatial Information Technology,42(03):43-46. 